\title{
Unserem Ehrenmitglied Prof. Friedrich-Ernst Stieve zum Geleit
}

Friedrich-Ernst Stieve wurde am 5. November 1915 in München als Sohn des späteren Professors für Anatomie in Halle/Saale und Berlin, Hermann Stieve, geboren. Auf seinen Vater geht die Umbenennung der Universität Halle/Saale in Martin-Luther-Universität zurück. Das in Tübingen begonnene Studium der Medizin schloss Friedrich-Ernst Stieve 1940 an der Humboldt-Universität Berlin mit einer Dissertation zum Thema „Nachbildung entfernter Menisken des menschlichen Kniegelenks“ ab. Im 2. Weltkrieg war er Militärarzt in Frankreich, Russland und Afrika und versorgte ab 1943 seine Mitgefangenen in amerikanischen Gefangenenlagern bis zu seiner Entlassung 1946. Im gleichen Jahr begann er als unbezahlter Volontär in der Röntgenabteilung des Städtischen Krankenhauses links der Isar in München und erlangte 1953 mit der Habilitationsschrift „Röntgenanatomische Studien zum normalen Schichtbild des Thorax und seiner Organe" die venia legendi an der Ludwig-Maximilians-Universität München.

Die radiologische Universitätsklinik, das „Rieder-Institut“, wurde seine langjährige Wirkungsstätte als akademischer Lehrer und praktisch und wissenschaftlich tätiger Radiologe, bis er 1975 die Leitung des „Instituts für Strahlenhygiene“ am Bundesgesundheitsamt in Berlin übernahm. Dieses war aus dem seit 1901 bestehenden traditionsreichen „Institut für Wasser-, Boden- und Lufthygiene“ des 1876 als Kaiserliches Gesundheitsamt gegründeten, späteren Bundesgesundheitsamtes hervorgegangen. Seine zentrale Aufgabe war die Forschung über die Wirkung von Radioaktivität auf den Menschen. Unter Friedrich-Ernst Stieve konnte 1980, kurz vor seiner Pensionierung im Alter von 65 Jahren, der Neubau des Instituts für Strahlenhygiene des Bundesgesundheitsamtes in Neuherberg bei München bezogen werden. Auch unter seinem Nachfolger Alexander Kaul entfaltete FriedrichErnst Stieve eine unermüdliche Tätigkeit zur Förderung des Strahlenschutzes. Er war Berater und Organisator von Konferenzen der Europäischen Kommission und von UNSCEAR in Wien und trug 1974 bis 1980 als Mitglied der deutschen Strah- lenschutzkommission mit seinem umfassenden Wissen zur Gestaltung der 1. deutschen Strahlenschutzverordnung bei. Seit 1989 ist das Institut für Strahlenhygiene Teil des nach der Tschernobyl-Katastrophe gegründeten Bundesamtes für Strahlenschutz und befasst sich u.a. mit der Analyse der natürlichen und zivilisatorischen Strahlenexposition, den radioökologischen Daten und Modellen, den somatischen und genetischen Wirkungen der Strahlenexposition, der Vorsorge für Störfälle und Unfälle und den Wirkungen nicht ionisierender Strahlen.

Das wissenschaftliche Lebenswerk von Prof. Stieve war 3 miteinander zusammenhängenden Themenkreisen gewidmet. Zum einen ging es um das Erkennen und Quantifizieren der Quellen der Strahlenexposition des Menschen. Wichtige Themen waren ihm die Organdosen bei radiologischen Untersuchungen aller Art, so auch die Erforschung der Organdosen bei Zufuhr radioaktiver Stoffe, z.B. beim diaplazentaren Übertritt. Seine Ziele waren die Reduktion der Organdosen bei Erhaltung der radiologischen Bildqualität und somit auch die Definition der Bildqualität und die Qualitätssicherung. An der Schaffung maßvoller Bestimmungen der Röntgenverordnung über die Aufzeichnung der zur Ermittlung der Organdosen geeigneten Daten, bei Vermeidung unnötigen Aufwands zur Ermittlung der Organdosen in jedem Einzelfall, war Prof. Stieve beteiligt. Mithilfe der WHO kam sein Engagement auch Entwicklungsländern zugute. Zum anderen ging es um die Ermittlung des mit Strahlenexpositionen verbundenen Gesundheitsrisikos, bis hin zu der schweren Aufgabe, Indikationskriterien für den Schwangerschaftsabbruch nach ungewollter oder unvermeidbarer Strahlenexposition aufzustellen.

Der 3., hieraus hervorgehende Aufgabenkreis betraf den praktischen Strahlenschutz, die vielfältigen Vorkehrungen und Maßnahmen zum Schutz des Menschen vor schädlichen Strahlenwirkungen. Prof. Stieve stellte sein Wirken unter den Grundsatz, dass jede medizinische Strahlenexposition durch ihren Nutzen für den Patienten gerechtfertigt sein muss und so

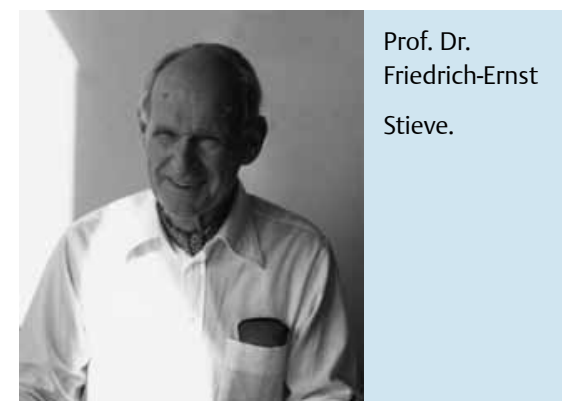

niedrig zu halten ist, wie sich das vernünftigerweise erreichen lässt. Dank seines wissenschaftlichen Ansehens konnte er dem Erlass der EURATOM-Richtlinien für den Strahlenschutz in der Medizin und der Einführung der „diagnostischen Referenzwerte“ als Mittel zur Optimierung des Strahlenschutzes des Patienten Nachdruck verleihen. Prof. Stieve erkannte die hohe Bedeutung einer guten Strahlenschutzausbildung der professionell Mitwirkenden und setzte sich in Kursen, durch seine Kurslehrbücher und als Verfasser von Richtlinien unermüdlich für die Fachkunde der Ärzte und die Ausbildung des medizinisch-technischen Personals im Strahlenschutz ein. Die Schlüsselstellung der Medizinphysiker für die Gewährleistung der Qualität der Strahlentherapie und der nuklearmedizinischen Diagnostik war der Ausgangspunkt für das entschlossene Eintreten von Prof. Stieve für die bestmögliche Gestaltung ihres Berufsbildes. Besondere Anliegen waren ihm die Weiterbildung der Medizinphysiker, ihre fachgerechte Integration in die Arbeit der radiologischen Kliniken und ihre verantwortliche Einbindung in die Qualitätssicherung in Diagnostik und Therapie. Eine weitere Brücke zur Physik schlug F.-E. Stieve in dem 1966 bis 1971 zusammen mit J. Grober herausgegebenen „Handbuch der Physikalischen Therapie“. Übereinstimmende Abschiedsworte haben die Unterzeichnenden Friedrich-Ernst Stieve daher auch in der „Zeitschrift für Medizinische Physik“ gewidmet.

Friedrich-Ernst Stieve wurde vielfach geehrt, darunter mit dem Verdienstkreuz 1. Klasse der Bundesrepublik Deutschland. Eine große Freude bereitete ihm die Berufung in die Leopoldina, die Nationale Akademie der Wissenschaften zu Halle, aus deren Mitgliedsliste das Porträt zu diesem Nachruf entnommen ist. Die Deutsche Röntgengesellschaft ernannte Prof. Stieve 1992 zu ihrem Ehrenmitglied. Das Bild von Friedrich-Ernst Stieve wäre aber unvollständig, wenn man schwere Stationen seines Lebens, wie den frühen Verlust 
seiner 1. Frau Eva und den seines Sohnes Joann, unerwähnt ließe. Im Rieder-Institut lernte er seine 2. Frau Rose Marie kennen, die ihn auf den Wanderungen seines Lebens begleitete, seine Enkel betreute und seinen Freunden eine liebenswerte Gastgeberin war. Das Bild von Friedrich-Ernst Stieve wäre auch unvollständig, wenn man seine Freude an Malerei, Musik und
Literatur unerwähnt ließe. Die sonntägliche Bachkantate im Radio gehörte dazu, und der Satz des Augustinus „Ama et fac, quod vis“ - „Liebe und tu, was du willst“ mag wohl ein Motto seines leidenschaftlichen Engagements und seiner gewaltigen Arbeitsleistung und Hilfsbereitschaft gewesen sein. Am 7. September 2012 starb Friedrich-Ernst Stieve nach einem der Wissenschaft und dem Strahlenschutz gewidmeten Leben. Seine Weggenossen und Schüler haben Prof. Stieve auf dem Münchner Waldfriedhof das letzte Geleit gegeben.

Dietrich Harder, Alexander Kaul und Fridtjof Nüsslin 